\title{
A Literature Review of Sensor Ontologies for Manufacturing Applications
}

\author{
Craig Schlenoff, Tsai Hong \\ Intelligent Systems Division, \\ National Institute of Standards and Technology \\ Gaithersburg, MD USA \\ craig.schlenoff@nist.gov, tsai.hong@nist.gov
}

Connie Liu

Dept. of Biomedical Informatics, Columbia University

New York, NY, USA

c12720@columbia.edu

\author{
Roger Eastman \\ Department of Computer Science \\ Loyola College of Maryland \\ Baltimore, MD USA \\ reastman@loyola.edu \\ Dr. Sebti Foufou \\ LE2i Lab, University of Burgundy \\ Dijon, France \\ Sebti.Foufou@u-bourgogne.fr
}

\begin{abstract}
The purpose of this paper is to review existing sensor and sensor network ontologies to understand whether they can be reused as a basis for a manufacturing perception sensor ontology, or if the existing ontologies hold lessons for the development of a new ontology. We develop an initial set of requirements that should apply to a manufacturing perception sensor ontology. These initial requirements are used in reviewing selected existing sensor ontologies. Additionally, we present our developed sensor ontology thus far that incorporates a refined list of requirements. This paper describes 1) extending and refining the requirements; 2) proposing hierarchical structures for verifying the purposes of the ontology; 3) choosing appropriate tools and languages (e.g., OWL (Web Ontology Language) [1] and SensorML (Sensor Markup Language) [2]) to support such an ontology; and 4) extending the Semantic Sensor Network (SSN) ontology with a set of refined requirements (i.e., our developed ontology). This work will be proposed as a standard within the IEEE (Institute of Electrical and Electronics Engineers) Robotics and Automation Society (RAS) Ontologies for Robotics Automation (ORA) Working Group [3] ${ }^{1}$.
\end{abstract}

Keywords-sensor ontology, OWL, literature review, manufacturing, ontologies for robotic and automation

\section{OVERVIEW OF THE SENSOR ONTOLOGY EFFORT}

Next generation robotic systems for manufacturing must perform highly complex tasks in dynamic environments. To improve return on investment, manufacturing robots and automation must become more flexible and adaptable, and less dependent on blind, repetitive motions in a structured, fixed environment. To become more adaptable, robots need precise sensing for parts and assemblies so they can focus on specific tasks in which they must interact with and manipulate objects. They also need situational awareness so they can robustly

\footnotetext{
${ }^{1}$ Certain commercial software and tools are identified in this paper in order to explain our research. Such identification does not imply recommendation or endorsement by the National Institute of Standards and Technology, nor does it imply that the software tools identified are necessarily the best available for the purpose.
}

sense their entire environment for long-term planning and short-term safety.

Meeting these requirements will need advances in sensing and perception systems that can identify and locate objects, can detect people and obstacles, and, in general, can perceive as many elements of the manufacturing environment as needed for operation. To robustly and accurately perceive many elements of the environment will require a wide range of collaborating smart sensors such as cameras, laser scanners, stereo cameras, and others. In many cases these sensors will need to be integrated into a distributed sensor network that offers extensive coverage of a manufacturing facility by sensors of complementary capabilities.

To support the development of these sensors and networks, the National Institute of Standards and Technology (NIST) manufacturing perception sensor ontology effort looks to create an ontology of sensors, sensor networks, sensor capabilities, environmental objects, and environmental conditions so as to better define and anticipate the wide range of perception systems needed. The ontology will include:

- detailed standard knowledge representations of sensor physical dimensions, weight, resolution, associated system performance, and operating conditions;

- representations of system capabilities, to categorize the functions that individual and groups of sensors can perform; and

- representations of sensor embeddings in sensor networks and the manufacturing environment.

The ontology will have three primary objectives. The first is to form the basis for new measurement methods to evaluate each perception system's (sensors and algorithms) ability to perform their required tasks. Prototypes of these perception systems are being developed, but science-based approaches to accurately and traceably measure their performance do not exist. The NIST sensor ontology effort is part of a larger project to develop the metrics and methods that underpin such 
approaches. Its focus will be the ability to detect people and objects as they move about the workspace. The project will build a testbed to conduct experiments to assess that ability. Project results will provide scientific foundations for new standards that enable the use of perception systems in manufacturing applications. One aspect of the testbed will be to test the system's ability to locate people. For this, the project will work to create ground truth for tracking the location of people, and metrics for evaluating systems against this ground truth. The sensor ontology will help to categorize sensors and their capabilities.

The second objective will be to support the design and calibration of networks by reasoning about required capabilities. By mapping sensors into capabilities and measured performance, the ontology will enable a facility designer to consider required capabilities at an abstract level and reason about the best performing and lowest cost combination of sensors to meet facility needs.

Similarly, the third objective is to enable an operating robotic system to reason about the available and running sensor systems to apply them to current perception needs, querying and retasking sensors as needed.

The purpose of this document is to review existing sensor and sensor network ontologies to understand whether they can be reused as a basis for a manufacturing perception sensor ontology, or if the existing ontologies hold lessons for the development of a new ontology. Before reviewing the existing ontologies for their application to manufacturing, this document states requirements that should apply to a manufacturing perception sensor ontology. Section 2 develops these requirements, Section 3 uses these requirements in reviewing selected existing sensor ontologies, and Section 4 presents our developed sensor ontology thus far that incorporates a refined list of the requirements. Subsequent documents will focus on a more detailed set of requirements for the manufacturing sensor ontology and the contents/structure of the manufacturing sensor ontology once it is fully developed.

\section{HIGH-LEVEL REQUIREMENTS FOR A MANUFACTURING SENSOR ONTOLOGY}

While sensors have similar characteristics across applications, from earth science to military to manufacturing, a sensor ontology for manufacturing will have unique needs and uses. Sensors will be (typically) installed in an indoor facility, used to perceive objects and manufacturing processes, designed to meet appropriate power and operating conditions, and repurposed as product lines undergo change. Key here are the required capabilities of a sensor to perceive objects and processes as distinct from other applications. In a Geographic Information System (GIS) earth science application, sensors usually measure basic physical properties (e.g., temperature, air pressure) meant to represent large areas, while in manufacturing a sensor may need to perceive the position and identity of relatively small parts. It may be possible to integrate sensor ontologies from different domains, but the integrated representation should be suitable in each. The intended purposes of the manufacturing perception sensor ontology are to:

- $\quad$ Provide a basis for measurement techniques to evaluate system performance.

- Support design of sensor networks for manufacturing applications.

- Categorize and organize calibration techniques for sensors and sensor networks.

- Reason about available sensors and capabilities to meet goals during operation.

- Create mechanisms and languages for querying sensors.

- Provide logs and data provenance to assess reliability, and for potential legal and regulatory review.

Based on these purposes, we can consider the sensor and system attributes that should be recorded in the ontology. Table 1 gives a potential list of attributes that could be associated with sensors and sensor systems. These are a high-level set of requirements and will be refined in subsequent phases of the effort.

The output of the sensors and sensor systems are important, and are described by five attributes in the table: Immediate data, Derived data, Algorithms, Integration/fusion, and Capabilities. Immediate data describes the raw, unfiltered data; Derived data describes data and interpretations computed from the original data; Algorithms describes the different, possibly alternative, procedures for computing Derived data; Integration/fusion describes derived data that comes from multiple sensor sources; and Capabilities describes the functional applications for which immediate and derived data can be used. It may be possible to collapse the five levels into fewer, but the current five are a working set of categories. It may be possible to organize data into a classic hierarchical model, such as raw data points, derived geometric entities like planes and surfaces, and semantic interpretations like objects and behaviors.

Table 1: General Attributes for Sensors and Sensor Systems/Networks

\begin{tabular}{|c|c|}
\hline Attributes & Comments \\
\hline Physical & Power, weight, size \\
\hline $\begin{array}{l}\text { Operating } \\
\text { conditions }\end{array}$ & Environmental conditions required for operation \\
\hline Immediate data & $\begin{array}{c}\text { Characteristics of data, resolution } \\
\text { (time/spatial/latency/frame rate/other) }\end{array}$ \\
\hline Derived data & $\begin{array}{l}\text { Results computed from raw data, both physical and } \\
\text { semantic }\end{array}$ \\
\hline Algorithms & Alternative algorithms for producing derived data \\
\hline $\begin{array}{l}\text { Integration/ } \\
\text { fusion }\end{array}$ & Data produced by combining data from multiple sensors \\
\hline Capabilities & Functional applications of raw and derived data \\
\hline Communication & Physical and logical protocols, and interoperability \\
\hline Processing & $\begin{array}{c}\text { On board processing power of sensors and network } \\
\text { nodes }\end{array}$ \\
\hline Calibration & $\begin{array}{l}\text { Individual and joint sensor calibration } \\
\text { information/algorithms }\end{array}$ \\
\hline Provenance & $\begin{array}{c}\text { Record of sensor and processing history of raw and } \\
\text { derived data }\end{array}$ \\
\hline Confidence & Levels of confidence in derived data \\
\hline
\end{tabular}


Algorithms are presented as a distinct attribute since a particular interpretation of the data, such as the presence of a person, may be computed in different ways from the raw data. For purposes of tracking provenance and confidence, it may be important to understand the processing steps that lead to the conclusion.

Capabilities are the interpretation, or affordances, of data as related to the goals of the overall robotic systems. The presence of a planar surface may be the derived data computed from a depth sensor; the fact that the surface affords a robot a platform for wheeled motion is a capability the sensor system provides the robot.

In addition to the concepts and attributes that must be supported by the ontology, a second question to answer is the nature of the ontology itself. What are the syntactic and semantic requirements for the ontology representation?

Given that sensor technologies are constantly changing, and that a manufacturing ontology should be applicable to different industrial domains, the ontology should be readably extensible. In particular, the capabilities that must be described will need to be adapted to the application domain. Based on the previously stated purposes for the manufacturing ontology, we know it should support reasoning with suitably complete and consistent descriptive logic. To describe dynamic environments and actions, the logic should manage temporal elements.

After further analysis of manufacturing scenarios that have a need for perceptions (such as those described in [4]), a slightly more refined set of concepts were discovered, as shown in Table 2. Some of these concepts are specializations of those presented in Table 1, while others are elaborations of the types of objects and interactions that must be perceived in the environment. More information about these requirements will be the topic of a future paper.

Table 2: Sensor Requirement Taxonomy

\begin{tabular}{|c|c|}
\hline Requirement Category & Example Requirements \\
\hline Domain & Bin Picking, Kitting, Assembly \\
\hline Object & $\begin{array}{c}\text { Kit, Part, Physical Barrier, Person, } \\
\text { Robot }\end{array}$ \\
\hline Group of Resources & Workcell, Flexible Assembly Line \\
\hline Robot Attribute & Working Volume \\
\hline Robot Component & Robot Arm, Perception System \\
\hline Robot Control/Motion Capability & $\begin{array}{c}\text { Follow A Person, Place Parts At A } \\
\text { Location, Stop At A Location, } \\
\text { Unload An Item }\end{array}$ \\
\hline Robot State & $\begin{array}{c}\text { Downtime, Halted, Normal } \\
\text { Operation }\end{array}$ \\
\hline Object Characteristics & Shiny, Flexible, Structured \\
\hline Perception System Capability & $\begin{array}{c}\text { Detect Human Pose, Track Moving } \\
\text { Object, Identify A Part }\end{array}$ \\
\hline Human State & Human Too Close, Human Contact \\
\hline Location & Work Area, Restricted Zone \\
\hline
\end{tabular}

\section{REVIEW OF SENSOR ONTOLOGIES}

In reviewing the literature on sensor ontologies, we selected seven research programs of interest and one review article. In this section we will look at each program and its relevance to the NIST sensor ontology project.

\section{A. Compton et al. Sensor Ontology Survey}

The survey article by Compton et al. [5] reviews 12 ontologies published from 2001 to 2009 . It looks at both the range and expressive power of the ontologies, as well as concepts these ontologies cannot describe accurately, with particular emphasis on sensor networks and the use of ontologies in their operation and description. Many existing sensor ontology articles, and the Compton et al. survey, are focused on the Semantic Web and the efforts to support autonomous agents that can find and use sensors attached to the Internet. This is a much larger and amorphous environment than a fixed manufacturing facility network, but similar concepts are needed for both.

\section{B. Ontology-Driven Adaptive Sensor Networks}

Avancha et al. [6] describe an ontology for adaptive sensor networks, with nodes that react to available power and environmental factors, calibrate for accuracy, and determine suitable operating states. The focus of this ontology is on adapting a wireless sensor network to current operating conditions while maintaining calibration and communication. The usefulness of this work for the NIST effort is in the concepts related to a sensor network and communication.

\section{A Formal Pedigree Ontology for Level-One Sensor Fusion}

Matheus et al. [7] developed an ontology that is intended for maintaining provenance of data from multiple sensors in a naval military application, so a user can understand how to judge and interpret sensor results. By associating the originating sensor output with data already represented in the ontology, the ontology can let users decide which of multiple sensor streams to trust. The usefulness of this work for the NIST effort is in the provenance concepts.

\section{An Ontology-Centric Approach to Sensor-Mission Assignment}

Gomez et al. [8] and Preece et al. [9] describe an ontology for automatically selecting sensors for task assignment based on sensor fitness for the task description. The system was designed to fulfill military missions given high-level descriptions of needed capabilities and available resources. The emphasis is on pre-mission planning, not during mission execution. The usefulness of this work is in the mission to sensor matching for system design.

\section{E. A Universal Ontology for Sensor Networks Data}

Eid et al. [10, 11] propose a two-tier framework for a sensor ontology. In their framework, the sensor hierarchy, data, and extension ontologies (lower tier) all reference the Standard Upper Merged Ontology (SUMO) (upper tier). SUMO [12] provides common high-level concepts that can be extended to produce domain specific ontologies, such as a sensor ontology. The lower tier ontology is divided into three parts: a Sensor Hierarchy Ontology ( $\mathrm{SHO}$ ) to describe transducer classes and attributes; a Sensor Data Ontology (SDO) to describe data; and a format for Extension Plug-in Ontologies (EPO) that allows developers to integrate domain-specific elements with the universal ontology. The usefulness of this article for the NIST 
effort is to provide a framework for integrating and extending ontologies, and using SUMO in a sensor ontology.

\section{F. The Semantic Sensor Network Ontology}

Neuhaus and Compton [13] discuss a generic ontology for describing sensors and deployments to be used in data integration, search, classification, and workflows. The ontology comprises four core clusters of concepts: (1) those concepts describing the domain of sensing (Feature); (2) those describing the sensor (Sensor); (3) those describing the physical components and location of the sensor (Sensor Grounding); and (4) those describing functions and processing (OperationModel and Process).

These clusters cover a wider range of concepts than the other ontologies. It is able to describe most of the spectrum of sensor concepts, including composition and structure. The ontology can describe more sophisticated forms of structural and sequencing composition, with, for example, sequence, conditional, and repetition for process composition. A combination of OntoSensor (described in the next section) and this ontology represents the current level of expressive capability for semantic sensors. However, currently this ontology cannot describe the components of platforms.

\section{G. Sensor Ontologies: From Shallow to Deep Models}

Russomanno et al. [14, 15] describe OntoSensor, an ontology intended as a general, knowledge base of sensors for query and inference. It is very general, and is comprised of definitions of concepts and properties adopted in part from SensorML [2] extensions to the Institute for Electrical and Electronics Engineers (IEEE) SUMO ontology [12], and constructs from the Web Ontology Language [1].

SensorML is a specification of a generic data model in Unified Modeling Language (UML) for capturing classes and associations that are common to all sensors. SensorML is part of an Open Geospatial Consortium (OGC) initiative to contribute to the development of a Sensor Web "through which applications and services will be able to access sensors of all types over the Web." [2]

OntoSensor extends the IEEE SUMO upper-level ontology by making some OntoSensor classes extensions of classes defined in SUMO. SensorML framework references some concepts that are defined in ISO 19115, which defines schema required for geographic information and services.

Other relevant projects and articles that have not been further explored due to the fact that they appeared too far outside of the scope of manufacturing include:

- The Coastal Environmental Sensing Networks (CESN) project at the University of Massachusetts (Boston) is developing technology for sensor networks for coastal observing and is described in [16].

- The OOSTethys community is developing open-source resources to help install, integrate, and update standards-compliant Web services for oceanographic observing, with a particular emphasis on OGC standards and is described in [17].

\section{H. IEEE 1451.4}

IEEE 1451.5 [18] is a standard for adding plug and play capabilities to analog transducers. While not an ontology per $s e$, it does provide a set of sensor-specific data requirements that can be used to build the ontology. The underlying mechanism for plug-and-play identification is the standardization of a Transducer Electronic Data Sheet (TEDS), which contains critical information needed by an instrument or measurement system to identify, characterize, interface, and properly use the signal from an analog sensor.

\section{The SSN Ontology}

A culmination of much of the work described above can be found in the Semantic Sensor Network (SSN) Ontology [19]. The W3C Semantic Sensor Network Incubator group (the SSN-XG) produced an OWL 2 ontology to describe sensors and their associated observations. The SSN ontology can describe sensors in terms of capabilities, measurement processes, observations, and deployments. It is organized, conceptually but not physically, into ten modules, as shown in Figure 1. The full ontology consists of 41 concepts and 39 object properties, directly inheriting from 11 DOLCE (Descriptive Ontology for Linguistic and Cognitive Engineering) concepts and 14 DOLCE object properties.

The ontology can describe sensors, the accuracy and capabilities of such sensors, observations, and methods used for sensing. Also, concepts for operating and survival ranges are included, as these are often part of a given specification for a sensor, along with its performance within those ranges. Finally, a structure for field deployments is included to describe deployment lifetime and sensing purpose of the deployed macro instrument.

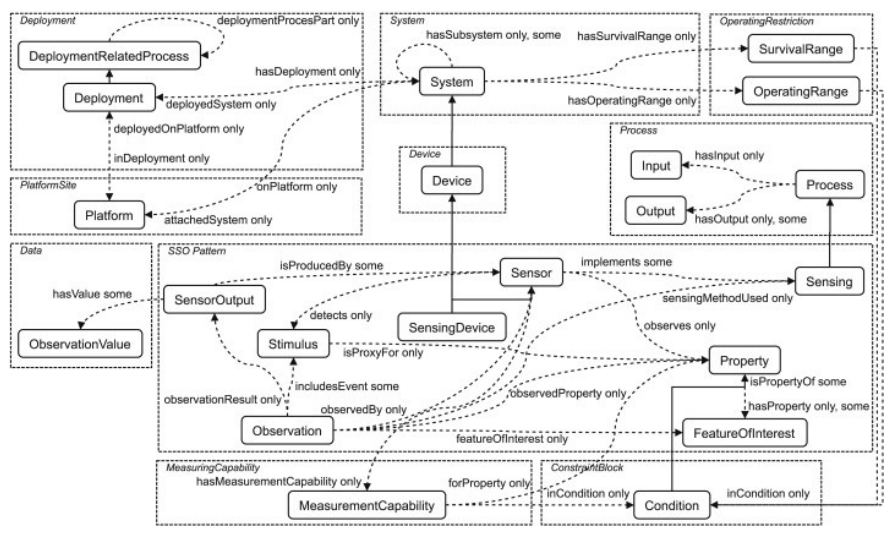

Figure 1: The SSN Ontology Structure

The SSN ontology is built around a central Ontology Design Pattern (ODP) [20] describing the relationships between sensors, stimulus, and observations. The ontology can be seen from four main perspectives:

- A sensor perspective, with a focus on what senses, how it senses, and what is sensed; 
- An observation perspective, with a focus on observation data and related metadata;

- A system perspective, with a focus on systems of sensors and deployments; and,

- A feature and property perspective, focusing on what senses a particular property or what observations have been made about a property.

The ontology takes a liberally inclusive view of what a sensor is: anything that observes; and allows such sensors to be described at any level of detail, for example, allowing sensors to be seen simply as objects that play a role of sensing, as well as allowing sensors to be described in terms of their components and method of operation. Humans and also simulations can be modeled as sensors.

\section{DEVELOPED SENSOR ONTOLOGY}

Based on our literature review of existing sensor ontologies and analysis of manufacturing scenarios with perception needs, we developed and refined a set of requirements for our ontology, as shown below. To implement the sensor ontology, we are extending the SSN ontology since it already describes sensors, measurement capabilities, properties, and more as aforementioned. Allow us to extrapolate on these additions made to the SSN ontology:

- Action: a relationship between objects where one acts upon another (e.g., robot performs an action on a part).

- Domain: an amalgamation of entities that belong to a particular manufacturing application. We have five domains: Assembly, Bin Picking, Kitting, Part Grasping, and Pick Up and Deliver Parts. In our ontology, these domains each have a list of constituents that are expected to be found in the domain. However, we leave it ultimately up to the end user to further define constituents, actions, intentions, and more depending upon the system being modeled.

- Additional Entities: additional types of objects have been added to the existing SSN ontology entity hierarchy to provide representation for different types of robots (e.g., water-based robot, air-based robot), abstract information (e.g., Manufacturer), physical artifacts (e.g., Container, Mechanical Component), physical places (e.g., end effector changing station), sensing devices (e.g., contact sensors, gas sensors), and systems (e.g., vision system).

- $\quad$ Resource Group: an object that represents a group of objects (i.e., a group of resources). The resource group object presents a unified architecture for representing and manipulating groups of resources, allowing them to be manipulated independently of the details of their representation. We have two types of resource groups, a List and a Mixed List. A List is a group of objects that have the same type and are sortable. A Mixed List is a group of objects that do not necessarily have the same type (e.g., a group of Part A, Robot B, and Workstation C). Resource Groups are useful in representing a group of objects, such as a kit tray, that a Robot may be manipulating.

- State: a representation of the current status of an Object. The value of a state of an object is one of a set of enumeration constants for that object type. We have four different types of states: State_Failure, State_HumanRobot, State_Part, and State_Workcell. State_Failure is the state of failure and applies to all entities. State_HumanRobot, State_Part, and State_Workcell apply to humans and robots, parts, and work cells, respectively. The State Object is useful for indicating the current status of an object and could be used in a reasoning system to track if a task has been completed or not.

- Intention: an aim or goal. This class serves as a way to indicate the intention of an entity. Our ontology does not specify the reasoning as to how to achieve the intention, and we leave that to the end user to define (since there are many different ways to achieve a certain intention depending on the system). However, we expect that to achieve an intention, there are a certain set of conditions that must be met and a series of actions or states that must be achieved in sequence.

- Physical Location: a reference point to a Physical Place entity (e.g., a Workcell). This allows for a user to have an object such as a Workstation act as the physical location of another Object (e.g., a Robot).

Besides these additions, we have also imported the Model Library for Quantities, Units, Dimensions, and Values ontology (QUDV) ${ }^{2}$. This allows us to assign properties that are quantities, such as length, mass, and more, consisting of a numerical value and a Unit object to our entities.

\section{SUMMARY}

Each of the ontologies described in Section 3 have elements useful in constructing a manufacturing perception sensor ontology. The three most general and comprehensive ontologies (as it pertains to the development of a manufacturing sensor ontology) are the Neuhaus/Compton, OntoSensor, and SSN Ontology efforts.

We have ultimately chosen to build from the SSN ontology. The list of requirements added to the ontology were developed and refined after reviewing the purposes of the ontology, reviewing the attributes in Section 2, coverage, and redundancy, analyzing perception needs of manufacturing scenarios, and putting the concepts into appropriate hierarchies.

The ontology was developed in the Web Ontology Language (OWL) as it is an extension of the SSN ontology, which is an OWL 2 ontology. The editing, development, and implementation were done using the open source ontology editor and knowledge-based framework Protégé ${ }^{3}$.

2 http://www.w3.org/2005/incubator/ssn/ssnx/qu/qu
3 http://protege.stanford edu 
After further refinement of our initial version of the manufacturing perception sensor ontology is completed, it will be proposed as a standard within the IEEE Robotics and Automation Society (RAS) Ontologies for Robotics Automation (ORA) Working Group [3]. The goal of this working group is to develop a standard ontology and associated methodology for knowledge representation and reasoning in robotics and automation, together with the representation of concepts in an initial set of application domains.

\section{REFERENCES}

F. Harmelen and D. McGuiness. (2004). OWL Web

Ontology Language Overview, W3C web site:

http://www.w3.org/TR/2004/REC-owl-features20040210\%.

[2] M. Botts and A. Robin, "Sensor Model Language (SensorML) implementation specification," 2007.

[3] C. Schlenoff, E. Prestes, R. Madhavan, P. Goncalves, H. Li, S. Balakirsky, et al., "An IEEE Standard Ontology for Robotics and Automation," presented at the International Conference on Intelligent Robots and Systems (IROS), Vilamoura, Algarve (Portugal), 2012.

[4] J. Shi, G. Jimmerson, T. Pearson, and R. Menassa, "Levels of Human and Robot Collaboration for Automotive Manufacturing," presented at the Performance Metrics for Intelligent Systems (PerMIS) Conference, College Park, MD, 2012.

[5] M. Compton, C. Henson, H. Neuhaus, L. Lefort, and A. Sheth, "A Survey of the Semantic Specification of Sensors," presented at the 2nd International Workshop on Semantic Sensor Networks, at 8th International Semantic Web Conference, 2009.

[6] A. Avancha, C. Patel, and A. Joshi, "Ontology-driven adaptive sensor networks," presented at the 1st Annual International Conference on Mobile and Ubiquitous Systems, Networking and Services, 2004. C. Matheus, D. Tribble, M. Kokar, M. Ceruti, and S. McGirr, "Towards a formal pedigree ontology for level-one sensor fusion," presented at the 10th International Command \& Control Research and Technology Symposium, 2005.

[8] M. Gomez, A. Preece, M. Plohnson, G. d. Mel, W. Vasconcelos, C. Gibson, et al., "An ontology-centric approach to sensor- mission assignment," presented at the 16th International Conference on Knowledge Engineering and Knowledge Management, 2008.

[9] A. Preece, M. Gomez, G. d. Mel, W. Vasconcelos, S. Sleeman, S. Colley, et al., "Matching sensors to missions using a knowledge-based approach," presented at the SPIE Defense Transformation and Net-Centric Systems, 2008.

[10] M. Eid, R. Liscano, and A. E. Saddik, "A novel ontology for sensor networks data," presented at the IEEE International Conference on Computational Intelligence for Measurement Systems and Applications, 2006.

[11] M. Eid, R. Liscano, and A. E. Saddik, "A universal ontology for sensor networks data," presented at the IEEE International Conference on Computational Intelligence for Measurement Systems and Applications, 2007.

[12] A. Pease, I. Miles, and J. Li, "The Suggested Upper Merged Ontology: A Large Ontology for Semantic Web and its Applications," in Working Notes of the AAAI-2002 Workshop on Ontologies and the Semantic Web, Edmonton, Canada, 2002.

[13] H. Neuhaus and M. Compton, "The semantic sensor network ontology: A generic language to describe sensor assets," presented at the AGILE Workshop: Challenges in Geospatial Data Harmonization, 2009.

[14] D. Russomanno, C. Korthari, and O. Thomas, "Building a sensor ontology: "A practical approach leveraging ISO and OGC models," presented at the 2005 International Conference on Artificial Intelligence, 2005.

[15] D. Russomanno, C. Kothari, and O. Thomas, "Sensor ontologies: from shallow to deep models," presented at the Thirty-Seventh Southeastern Symposium on System Theory, 2005.

[16] T. D. C. Little, J. Knorad, and P. Ishwar, "A Wireless Video Sensor Network for Autonomous Coastal Sensing," presented at the Conference on Coastal Environmental Sensing Networks (CESN), 2007.

[17] E. Bridger, L. E. Bermudez, M. Maskey, C. Rueda, B. L. Babin, and R. Blair, "OOSTethys - Open Source Software for the Global Earth Observing Systems of Systems," presented at the American Geophysical Union, Fall Meeting 2009.

[18] IEEE, "IEEE standard for a smart transducer interface for sensors and actuators wireless communication protocols and transducer electronic data sheet (TEDs) formats - IEEE Std 1451.5," ed, 2007, pp. C1 - 236.

[19] M. Compton, P. Barnaghi, L. Bermudez, U. GarciaCastro, O. Corcho, S. Cox, et al., "The SSN Ontology of the W3C Semantic Sensor Network Incubator Group," Web Semantics: Science, Services and Agents on the World Wide Web, vol. 17, pp. 25-32, 2012.

[20] A. Gangemi, "Ontology Design Patterns for Semantic Web Content," presented at the 4th International Semantic Web Conference, Lecture Notes in Computer Science, 2006. 